\title{
Habituation of open-field escape responses and increase in competing responses in the Mongolian gerbil, Meriones unguiculatus
}

\author{
HAROLD R. BAUER \\ Boys Town Institute for Communication, Omaha, Nebraska 68131 \\ and \\ PHILIP H. GRAY \\ Montana State University, Bozeman, Montana 59717

\begin{abstract}
The relationship between open-field escape responses to visual stimuli over repeated daily exposures and the frequency of ambulation, territorial marking, autogrooming, digging, and time out of the field were studied in the Mongolian gerbil, Meriones unguiculatus. Startle and escape responses declined over 6 days of repeated exposures, whereas the initially suppressed levels of competing responses increased.
\end{abstract}

Of all behavioral changes that have been termed learning, the habituation process is recognized as being one of the most common through ontogeny and in different phyla (Hinde, 1970; Lorenz, 1965; Marler \& Hamilton, 1966; Ratner, 1970; Thorpe, 1965). Thorpe (1965) refers to habituation "in the strict sense" as longtime stimulus-specific response decrement, although he extends the use of the term to "the waning of a response as a result of repeated stimulation that is not followed by any kind of reinforcement" (p.487). Habituation is defined here as a response decrement occurring as a behavioral adaptation to a repeated stimulus situation that has no further consequences (Gray, 1966a, p. 34), but not due to a neuronal refractory period, sensory adaptation, or effector fatigue (Hinde, 1970, p. 3).

We studied an open-field escape response in the Mongolian gerbil (Meriones unguiculatus), which is a colonial burrow-living rodent that constructs nests and storerooms in dry sandy soil and uses the burrows to escape from predators on the flat, open surface of fields (Tanimoto, 1943). The aims of the investigation were to examine the escape response to fast-moving overhead stimuli, response habituation occurring over days, and the relationship between the escape response and other ongoing behaviors, such as digging, territorial marking, self-grooming, and locomotion. A review of the origin and development of studies of aerial predator reactions has been done by Gray (1966b).

\section{METHOD}

Twenty-seven Mongolian gerbils (Meriones unguiculatus), 13 males and 14 females, were used at approximately 180 days of

This research was done while both authors were with the Department of Psychology, University of Manitoba at Winnipeg, and was supported by a Faculty Research Grant. Requests for reprints may be sent to either author. age at initial testing. The gerbils were purchased from Tumblebrook Farms, Inc. They were housed as mated pairs in a whitenoise-masked 12-h light/dark cycle animal room.

The apparatus consisted of a 1-m-square open field with a grid of 16 equally sized squares drawn in black on a white semigloss floor. The field was studded with nine frosted Plexiglas pegs measuring $25 \mathrm{~mm}$ long, $11 \mathrm{~mm}$ wide, and $7 \mathrm{~mm}$ high, after Thiessen (1968). Each peg was positioned for use as a territorial marking site at each of the nine intersecting points of the grid. Three of the $122 \times 122 \mathrm{~cm}$ white semiglossed Masonite sides to the open field met with the fourth wall, which was $36 \mathrm{~cm}$ high and consisted of the lower $22 \mathrm{~cm}$ of white Masonite topped by $14 \mathrm{~cm}$ of clear Plexiglas. The Plexiglas top permitted the predator stimulus to be viewed from the opposite $75 \%$ of the field. Twenty centimeters in the back of the fourth wall, a white semiglossed $122 \times 122 \mathrm{~cm}$ cardboard partition provided a blind for the visual stimulus launching apparatus and the observer. The visual stimulus moved through a $6.5 \times 40.5 \mathrm{~cm}$ horizontal slot $20 \mathrm{~cm}$ from the floor base of the blind, and the observer had a $6.5 \times 19 \mathrm{~cm}$ horizontal slot adjacent to the launching apparatus in the blind. A darkened room and a fluorescent light over the open field permitted only limited acuity by the gerbils through the blind.

The visual stimulus was a reversible, black, long-necked (goose-like) or short-necked (hawk-like) form, after Schleidt (1961). The stimulus was $35.5 \mathrm{~cm}$ wide $\times 30.5 \mathrm{~cm}$ long and moved on the end of a $150 \mathrm{~cm}$ long $x 2 \mathrm{~cm}$ square white pole, attached to a curtain track driven by a reversible electric motor so that it could move horizontally $45 \mathrm{~cm}$ off the floor to the opposite side of the open field, coming to a halt over the opening of the enclosure. The automatic ejection of the stimulus into the open field was at a fixed rate of $122 \mathrm{~cm} / \mathrm{sec}$, with a reduced recall speed.

In the center, at the base of the wall opposite to the entrance for the visual stimulus, a $15 \mathrm{~cm}$ long $\times 6.5 \mathrm{~cm}$ square "burrow" enclosure was placed. This enclosed area consisted of only $1 \%$ of the total apparatus area open to the gerbils and had white flooring with black top and sides.

A 20-min exposure to the open field was used for each gerbil. Three groups of nine gerbils matched for sex but otherwise randomly distributed were exposed to the open field one at a time, for $20 \mathrm{~min} /$ day, for 7 consecutive days. On the $1 \mathrm{st}$ day of exposure to the field, all animals were permitted to move freely and explore their new environment while being observed. No presentation of the visual stimulus was made to any of the three groups on the 1 st day. 
The frequency and temporal distribution of various behaviors in 1-min blocks were recorded on the data sheet. With the exceptions of territorial marking and line crossing, these behaviors were scored as having occurred, or not occurred, in each 1-min block. The frequency of territorial marking was the only behavior for which each occurrence per 1-min block was scored. The frequency of line crossing was summed for the entire 20-min session and each crossing was counted. The behavioral classes included digging (a rapid movement of the forepaws against an object), washing (a care of the body surface with the mouth), forepaw wipe (a care of the body surface by sweeping the forepaws over the pelage). The amount of observed time that was spent out of the field and the number of exits from it were recorded on the clock and counter over the entire 20-min session.

The 1st day in the field was without stimulus presentation. The 2 nd day of experimentation, four male and five female gerbils in Group A received eight presentations of the shortnecked (hawk-like) stimulus during the last $15 \mathrm{~min}$ of the 20-min session. Both the startle response and the open-field escape response were scored as having occurred or not occurred for each presentation on the data sheet. The startle response was defined as immediate rapid flight and orientation from the stimulus source. The open-field escape response was defined as such rapid flight followed by leaving the open field within $10 \mathrm{sec}$ of the launching of the stimulus. All stimulus presentations were made while the anterior of the animal faced the stimulus source at less than $90 \mathrm{deg}$ from its sagittal plane in the opposite $75 \%$ of the open field. The response was scored only as having occurred or not. This procedure was followed from the 2 nd to the 6 th day in Group A. On the 7 th day, the stimulus was reversed to the long-necked stimulus for the first four of eight presentations. The identical procedure was followed for Group B, except that its four males and five females received the long-necked (goose-like) stimulus for Days 2-6 and were counterbalanced on Day 7, receiving four short-necked and four longnecked stimulus presentations. In this manner, both experimental groups received the same procedure, with the exception of having the opposite stimuli. Basal observations were made on the five males and four females of Group C, which served as a control group in that no visual stimuli were presented on Days 1-7.

Appropriate nonparametric statistical analyses were carried out as suggested by Siegel (1956).

\section{RESULTS}

The startle and the open-field escape responses in the experimental groups occurred significantly more than in the control group from Day 2 to Day 7 [Wilcoxon $\mathrm{T}(5)=0, \mathrm{p}<.01]$ (Figure 1a). Initial responses to the experimental stimulus included "foot thumping" and audible, repetitious, and simultaneous stamping of the hindlimbs (Spatz \& Granger, 1970). Foot thumping in the gerbil colony room often led to several seconds of visually separate gerbils freezing in an extended upright position and a "testing of the air."

The number of line crossings as an index of ambulation for the three groups is shown in Figure 1b. The difference in the ambulation between Group A and the control group was significant on Day 2, the 1st day of stimulus presentation $[\mathrm{T}(8)=4, \mathrm{p}<.05]$. The same was the case for the differences in ambulation between Group B and the control group $[\mathrm{T}(8)=0, \mathrm{p}<.01]$. From Day 2 to Day 3, an increase in ambulation occurred for both experimental groups and a corresponding

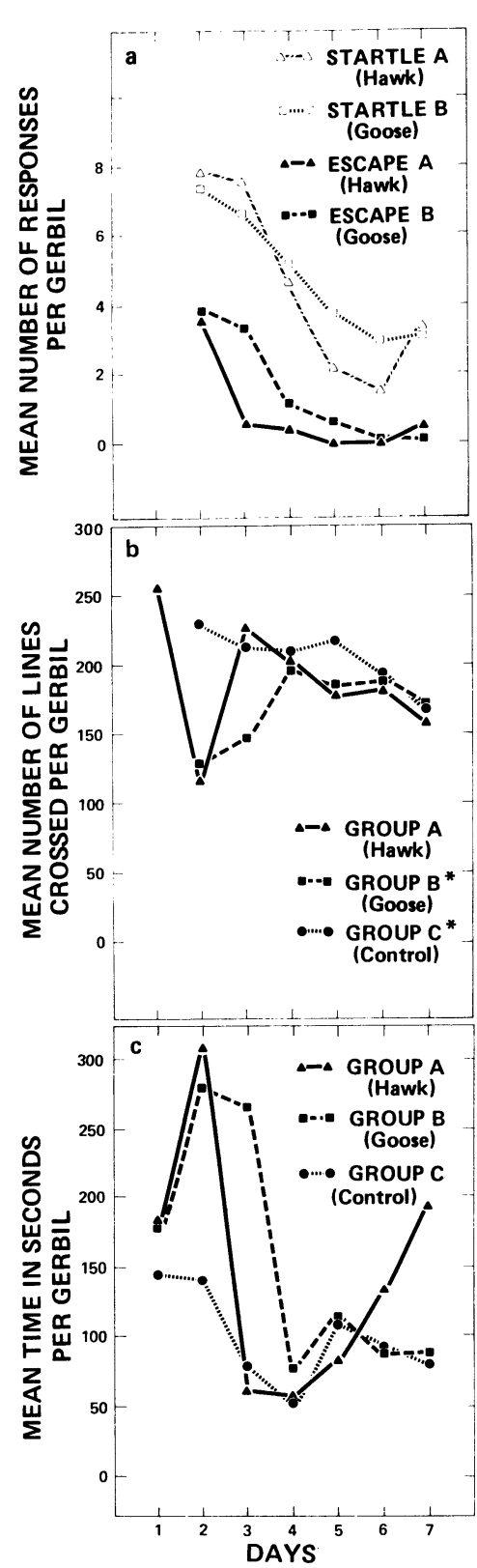

Figure 1. (a) Startle and escape response decrement to hawk-like (Group A) and goose-like (Group B) stimuli; (b) ambulation, showing initial decrement and subsequent replacement in experimental groups; (c) time in enclosure, showing initial increase with stimulus presentation and subsequent decrement in experimental groups. In all cases, Day 1 of the experimental groups and all days of the control group had no stimulus presentations and Day 7 of the experimental groups had counterbalanced stimuli.

decrease occurred for the control group to a point at which there was no significant difference between them on Day $3(\mathrm{p}<.05)$.

An increase in the amount of time spent out of the open field occurred in the two experimental groups, although neither difference was significant (Figure 1c). The drop in the time spent in the enclosure from Day 2 
to Day 3 in Group A was significant $[\mathrm{T}(8)=4, \mathrm{p}<.05]$. A sex difference was found for the time spent in the enclosure in both experimental groups. In each of the two groups, in which 48 stimulus presentations were given over the last 6 days, females spent more time in the enclosure than males did (Table 1). In the control group, the relationship was reversed $(p>.05)$.

Territorial marking was another dependent measure that declined in occurrence in the two experimental groups after the onset of the stimulus presentation. The differences shown in Figure 2a between the malcs in Group A and Group C on Days 2 and 3 were significant according to the Mann-Whitney $U$ test $(U=0, p<.014)$. The males in Group B were only significantly different from Group C on Day $3(\mathrm{U}=0, \mathrm{p}<.014)$. Overall sex differences were apparent, with males marking 13.7 times more than females, a finding consistent with the reports of sex differences by Thiessen (1968).

Other behaviors whose changes in frequency coincide in the experimental groups with the onset of the stimulus presentation on Day 2 are digging and the two forms of grooming: the forepaw wipe and the body wash. Digging was observed to increase from Day 1 to Day 2 in the control group and decreased in the experimental groups (Figure 2b). The differences, however, were significant only between Group $B$ and the control group (C) on Days 2 and $3[\mathrm{~T}(8)=3, \mathrm{p}<.02 ; \mathrm{T}(8)=$ $4.5, \mathrm{p}<.05]$. Overall, it would be conservative to say that $75 \%$ of the digging occurred in the enclosure (i.e., $1 \%$ of the total area open to the gerbils).

A sharp increase in forepaw wiping and washing occurred in the control subjects (Group C), as shown by Figure $2 c$, with a corresponding decrease from Day 1 to Day 2 in the experimental subjects (Groups A and B). Group A was significantly lower in forepaw wiping and washing on Day 2, although not on Day $3[\mathrm{~T}(8)=0$, $p<.01]$. Group B had significantly fewer wipes and washes compared with the control group on Days 2 and $3[\mathrm{~T}(5)=3, \mathrm{p}<.02 ; \mathrm{T}(5)=4, \mathrm{p}<.05]$. Body washing was a longer and less frequently occurring form of grooming compared with the briefer forepaw wipe.

Counterbalancing on the 7 th day by presenting the goose-shaped stimulus for the first four trials to Group A and the hawk-shaped stimulus for the first four trials to Group B resulted in differences between groups in responsivity, as shown in Figure 1a. Group A made a significant increase in startle responses from Day 6 to Day 7 as well as significantly more responses for the new goose-shaped stimulus.

Table 1

Mean Time in the Enclosure per Daily Session

\begin{tabular}{crr}
\hline Group & Male & Female \\
\hline $\mathrm{A}^{*}$ & 114.4 & 173.5 \\
$\mathrm{~B} \dagger$ & 98.0 & 206.6 \\
$\mathrm{C}$ & 124.9 & 84.0 \\
\hline
\end{tabular}

*Significant sex differences $(U=3, p<.056)$.

†Significant sex differences $(U=2, p<.032)$.

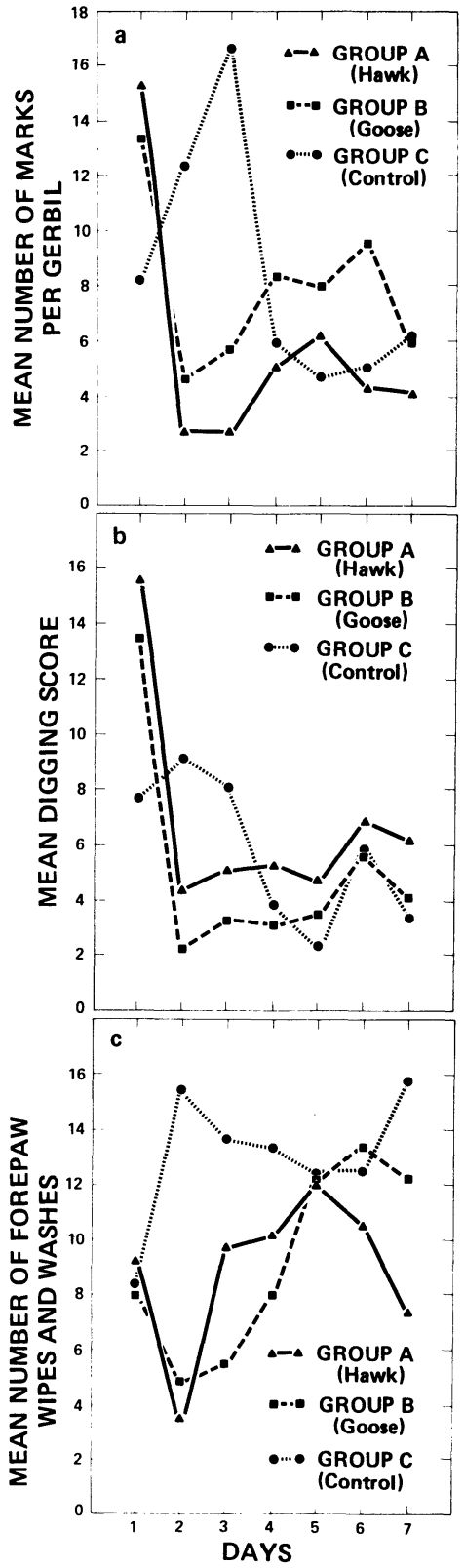

Figure 2. (a) Territorial marking, showing initial increase in control group and initial decrease and subsequent replacement in experimental groups; (b) digging, showing initial increase in the control group and initial decrease and subsequent replacement in the experimental groups; (c) self-grooming, showing initial increase in the control group and initial decrease and subsequent replacement in experimental groups. In all cases, Day 1 of the experimental groups and all days of the control group had no stimulus presentations and Day 7 of the experimental groups had counterbalanced stimuli.

\section{DISCUSSION}

The open-field responses (startle and escape) appear to be different forms of the same flight responses to looming visual stimuli. The escape response's "burrow flight" may be an apparatus-related result (e.g, a slippery surface led to sliding) of ten repeated in later trials (Figure 1a). Gerbils finding the 
burrow may have habituated slower than the gerbils using the startle response, due to less stimulus exposure time (Hoffman \& Ison, 1980).

Initially, the experimental stimuli reduced gradual exploration, territorial marking, digging, and forepaw wipe and body washing (Figures $1 \mathrm{~b}, 2 \mathrm{a}, 2 \mathrm{~b}$, and $2 \mathrm{c}$ ), while inducing flight followed by freezing. As habituation to the experimental stimuli took place, the competing responses of ambulation, territorial marking, digging, and forepaw wipe and body wash "replaced" the experimentally induced startle and escape responses (Ratner, 1970). These data are experimental evidence supporting the notion that habituation permits the waning of biologically irrelevant responses to insignificant stimuli in favor of biologically relevant behaviors (Lorenz, 1965; Marler \& Hamilton, 1966).

Sex differences in an antipredator response rather than "burrow time" are suggested by both experimental groups of females spending more time out of the open field than males, since the control group had the opposite relationship, with the male spending more time in the burrow (Table 1). Females tended to be more reactive than males to the experimental stimuli $(\mathrm{p}>.05)$. The burrow was initially used by experimental subjects to avoid the looming stimuli (Figure 1c) on the first four trials of Day $7[\mathrm{~T}(8)=0, \mathrm{p}<.05 ; \mathrm{T}(8)=0, \mathrm{p}<.01]$. Open-field escape responses in both groups occurred to the counterbalanced stimulus but were nonsignificant $(p>.05)$. The time spent in the enclosure on the counterbalanced day for Group A increased considerably (Figure 1c). The frequency of forepaw wipes and body washes decreased and was significantly different from controls on Day $7[\mathrm{~T}(8)=0, \mathrm{p}<.01]$. No overall differences in the frequency of the open-field escape response between Groups A and B were found between Days 2 and 6 (Friedman two-way ANOVA, $\mathrm{p}>.05$ ).

After startle and open-field escape responses ceased occurring, changes in behavior upon presentation of the stimulus were still most often noticeable, with subjects responding with head orientation to the stimulus in most cases. Approach responses from a standing position in the direction of the stimulus entrance window followed by orientation toward the window in the upright position were common. In some cases, an extended upright position was common. In some cases, an extended upright position and "testing the air" occurred while the animal was oriented toward the stimulus entrance, consummating the approach sequence.

The temporal relationship between previous locomotion and escape responses may be a factor in the rate of habituation (Fentress, 1968; Hoffman \& Ison, 1980). Fentress (1968) found that the probability of wild-trapped voles (Microtus agretis and Clethroinomys britanicus) fleeing an overhead visual stimulus was much greater if locomotion either was concurrent or had ceased within $10 \mathrm{sec}$. Vole responses differed from those of the Mongolian gerbil, since all the voles exhibited some initial freez- ing, whereas the flight was immediate in the gerbil. A limited understanding exists of the neurobiological mechanisms underlying species differences in responsiveness that may be a result of ecological adaptation of the flight behavior (Bolles, 1970).

\section{REFERENCES}

Bolles, R. C. Species-specific defense reactions and avoidance learning. Psychological Review, 1970, 77, 32-48.

Fentress, J. C. Interrupted ongoing behaviour in two species of vole (Microtus agretis and Clethroinomys britanicus). II. Extended analysis of motivational variables, underlying fleeing and grooming behaviour. Animal Behaviour, 1968, 16, 154-167.

Gray, P. H. The comparative analysis of behavior. Dubuque, Iowa: Brown, 1966. (a)

Gray, P. H. Historical notes on the aerial predator reaction and the Tinbergen hypothesis. Journal of the History of the Behavioral Sciences, 1966, 2, 330-334. (b)

Hinde, R. A. Animal behavior: A synthesis of ethology and comparative psychology (2nd ed.). New York: McGraw-Hill, 1970.

Hoffman, H. S., \& Ison, J. R. Reflex modification on the domain of startle: I. Some empirical findings for how the nervous system processes sensory input. Psychological Review, 1980, 87, 175-189.

LoREnz, K. Evolution and modification of behavior. Chicago: Chicago University Press, 1965.

Marle R, P., \& Hamilton, W. H. Mechanisms of animal behavior. New York: Wiley, 1966.

RAtner, S. C. Habituation: Research and theory. In J. H. Reynierse (Ed.), Issues in animal learning. Lincoln: University of Nebraska Press, 1970.

SchleIDT, M. Über die Auslösun der Flucht vor Rabuvögeln bei Truthühnern. Naturwissenschaftern, 1961, 48, 141-142.

Siegel, S. Nonparametric statistics for the behavioral sciences. New York: McGraw-Hill, 1956.

Spatz, C., \& Granger, W. R. Foot-thumping in the gerbil: The effect of establishing a home cage. Psychonomic Science, 1970, 19, 53-54.

TANimoto, $\mathbf{K}$. Ecological studies of plague-carrying animals in Manchuria. Dobutsugaku Zasshi, 1943, 55, 11-127.

Thiessen, D. D. The roots of territorial marking in the Mongolian gerbil: A problem of species-common topography. Behavior Research Methods \& Instrumentation, 1968, 1, 70-76.

Thorpe, W. H. The ontogeny of behavior. In J. A. Moore (Ed.), fteas in modern biology. Garden City, New York: Natural History Press, 1965.

(Received for publication July 13, 1981,) 